\title{
Transplanted endothelial cells repopulate the liver endothelium and correct the phenotype of hemophilia A mice
}

\author{
Antonia Follenzi, ${ }^{1,2}$ Daniel Benten, ${ }^{3}$ Phyllis Novikoff, ${ }^{1}$ \\ Louisa Faulkner, ${ }^{4}$ Sanj Raut, ${ }^{4}$ and Sanjeev Gupta1,3,5 \\ ${ }^{1}$ Department of Pathology, Marion Bessin Liver Research Center, Albert Einstein College of Medicine, New York, New York, USA. \\ 2Department of Biomedical Sciences, University of Piemonte Orientale, Novara, Italy. ${ }^{3}$ Department of Medicine, Albert Einstein College of Medicine, New York, \\ New York, USA. ${ }^{4}$ Haemostasis Section, Biotherapeutics Group, National Institute for Biological Standards and Control, Hertfordshire, United Kingdom. \\ ${ }^{5}$ Cancer Research Center and Institute for Clinical and Translational Research, Albert Einstein College of Medicine, New York, New York, USA.
}

\begin{abstract}
Transplantation of healthy cells to repair organ damage or replace deficient functions constitutes a major goal of cell therapy. However, the mechanisms by which transplanted cells engraft, proliferate, and function remain unknown. To investigate whether host liver sinusoidal endothelium could be replaced with transplanted liver sinusoidal endothelial cells, we developed an animal model of tissue replacement that utilized a genetic system to identify transplanted cells and induced host-cell perturbations to confer a proliferative advantage to transplanted cells. Under these experimental conditions, transplanted cells engrafted efficiently and proliferated to replace substantial portions of the liver endothelium. Tissue studies demonstrated that transplanted cells became integral to the liver structure and reacquired characteristic endothelial morphology. Characterization of transplanted endothelial cells by membrane markers and studies of cellular function, including synthesis and release of coagulation factor VIII, demonstrated that transplanted cells were functionally intact. Further analysis showed that repopulation of the livers of mice that model hemophilia A with healthy endothelial cells restored plasma factor VIII activity and corrected their bleeding phenotype. Our studies therefore suggest that transplantation of healthy endothelial cells should be considered for cell therapy of relevant disorders and that endothelial reconstitution with transplanted cells may offer an excellent paradigm for defining organspecific pathophysiological mechanisms.
\end{abstract}

\section{Introduction}

A major requirement of cell therapy concerns reconstitution of specific cell compartments. Recently, several studies established the possibility of replacing parenchymal liver cells with transplanted hepatic or extrahepatic donor-derived cells (1). Similarly, replacement of ECs in specific vascular beds, such as the liver, would be a profoundly significant accomplishment because ECs play central roles in liver development, organization, repair, and function. Moreover, ECs synthesize critical coagulation factors and contribute to cell-cell signaling or immunoregulation (2-5). Therefore, the ability to reconstitute the endothelium of organs with healthy cells will offer therapeutic opportunities, and organs other than the liver will also be suitable targets for this purpose, e.g., to restore cardiovascular or pulmonary functions $(6,7)$. At present, differences in the properties of organ-specific ECs are not fully understood. The inability of i.v.-injected pulmonary ECs to target extrapulmonary organs, e.g., the liver or spleen, and of liver sinusoidal ECs (LSECs) to target extrahepatic organs, e.g., lungs, heart, or kidneys, indicates that cell and organ type-specific differences in EC populations need to be further defined $(7,8)$.

Nonstandard abbreviations used: DiI-Ac-LDL, 1,1-dioctadecyl-3,3,3,3-tetramethylindocarbocyanine-labeled acetylated LDL; FIX, coagulation factor IX; FLP, fibronectin-like polymer; FVIII, coagulation factor VIII; LSEC, liver sinusoidal EC; MCT, monocrotaline; NPC, nonparenchymal cell; SDF-1, stromal cell-derived factor-1; SEM, scanning electron microscopy; TEM, transmission electron microscopy. Conflict of interest: The authors have declared that no conflict of interest exists. Citation for this article: J. Clin. Invest. 118:935-945 (2008). doi:10.1172/JCI32748.
To understand mechanisms in reconstitution of the hepatic endothelium, we developed robust transplantation assays with transgenic donor cells and manipulated recipients prior to cell transplantation for enhancing cell engraftment and proliferation. Similarly, model systems are needed to effectively demonstrate gain of function. Here, we addressed the role of transplanted LSECs in synthesizing and releasing the coagulation protein factor VIII (FVIII). This essential coagulation factor is lacking in hemophilia A, which has a prevalence of 1 in 10,000 males and is characterized by recurrent, spontaneous bleeding leading to disability or fatality (9). Hemophilia A is an attractive target for cell and gene therapy, as maintaining FVIII levels in the $1 \%-5 \%$ range decreases the risk of spontaneous bleeding. Infusion of recombinant clotting factor is effective in the short term for preventing or treating bleeding in people with hemophilia, although prevention of bleeding complications in the long term requires multiple weekly infusions, which are inconvenient, invasive, and costly. Also, generally up to $25 \%$ of hemophilia A patients develop inhibitory antibodies against FVIII clotting factors that may render further replacement therapy ineffective (10). Despite insights into genetic defects in hemophilia A, the precise organ- and cell type-specific origins of FVIII have been controversial $(2,11,12)$. As LSECs produce FVIII, we addressed the therapeutic potential of liver repopulation with transplanted LSECs in hemophilia A mice. Our studies indicate that transplanted cells have potential for future clinical applications. Moreover, these studies offer suitable directions to approach restoration of additional vascular compartments with transplanted ECs. 
A

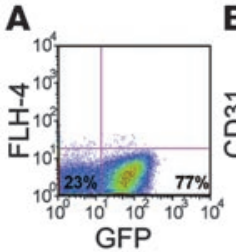

$\mathbf{F}$

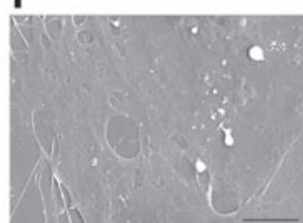

I

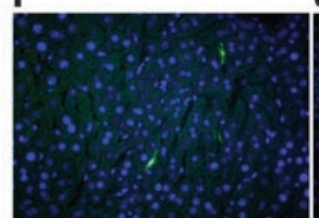

$\mathbf{L}$

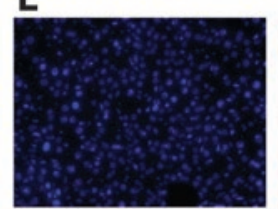

$\mathbf{P}$

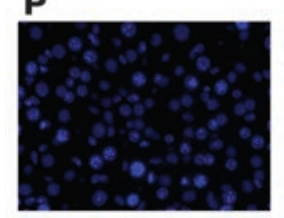

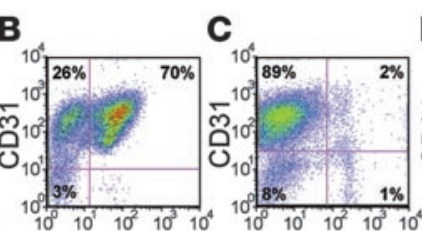

GFP

G

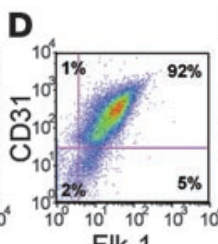
H

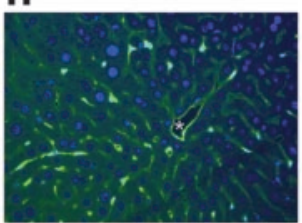

K

K

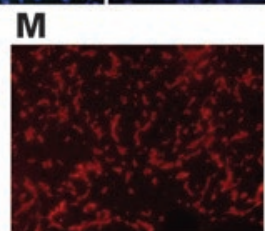

Q

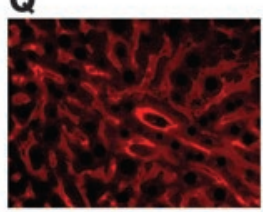

E

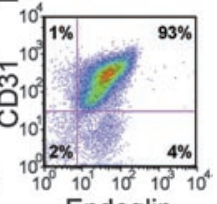

Endoglin

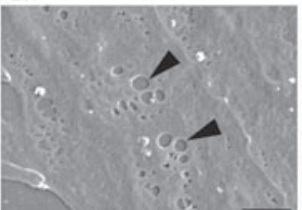

J

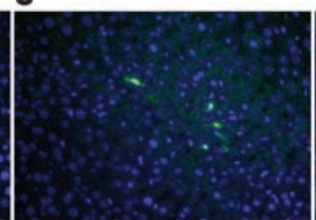

$\mathbf{N}$

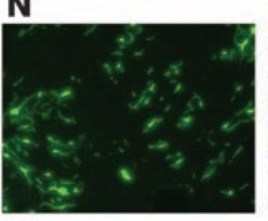

R

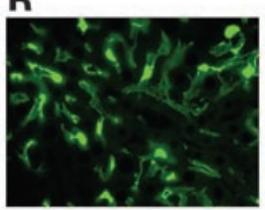

0

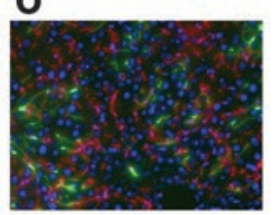

s

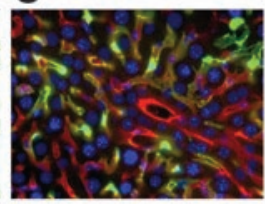

\section{Figure 1}

Properties of isolated FVB/N-Tie2-GFP LSECs and fate of transplanted LSECs. Flow cytometry showing GFP-positive LSECs selected with anti-LSECs after CD45-positive cells were depleted. LSECs selected showed native GFP expression in $77 \%$ (A); 96\% stained for the endothelial marker CD31 (B), and only 3\% stained for CD45 (C). Moreover, CD31-containing LSECs coexpressed additional endothelial markers, Flk-1 (D) and endoglin (E). (F and G) SEM showing sieve plates with fenestrae on LSEC surface (arrowheads), another feature of sinusoidal ECs. (H) Donor FVB/N-Tie2-GFP mouse liver showing GFP staining (green) in LSECs, along with a portal vein radicle (asterisk). (I-K) FVB/N mice after LSEC transplantation. (I) Two transplanted cells identified by GFP staining (green) in liver sinusoids. (J) Increased engraftment of transplanted LSECs 1 week after cell transplantation in FLP-treated mouse. (K) Significantly increased engraftment of transplanted LSECs 1 week after cell transplantation in MCT-treated mouse. (L-S) GFP fluorescence in transplanted LSECs to indicate cell proliferation 3 months after transplantation in MCT-treated mice. ( $\mathbf{L}$ and $\mathbf{P})$ Nuclear staining with DAPI (blue). (M) Kupffer cells immunostained with F480 antibody (red). (Q) Endothelial cells immunostained with CD31 antibody. (N and R) GFP immunostaining. ( $\mathbf{O}$ and $\mathbf{S}$ ) Merged images from all 3 panels. Original magnification, $\times 2,500(F)$; $\times 12,500$ (G); ×200 (H-K); ×400 (P-S); ×200 (L-O). Scale bars: $2 \mu \mathrm{m}(\mathbf{F}) ; 0.4 \mu \mathrm{m}(\mathbf{G})$.

\section{Results}

Properties of isolated FVB/N-Tie2-GFP LSECs. To identify transplanted ECs in recipients, we used transgenic FVB/N-Tie2-GFP mice as cell donors because only ECs would express GFP under transcriptional control of the endothelial receptor tyrosine kinase promoter Tie2 (13). This GFP reporter helped in identifying LSECs during their characterization in vitro. Flow cytometric analysis, including Tie2-driven GFP expression and EC membrane markers, e.g., PECAM-1 (CD31), endoglin (CD105), Flk-1, and Tie2, indicated that isolated LSECs possessed endothelial characteristics (Figure 1, A-E, and not shown). These cells showed infrequent contaminants with $3 \% \mathrm{CD}^{+} 5^{+}$cells (Figure 1C); within this $\mathrm{CD}^{2} 5^{+}$fraction, the majority were $\mathrm{CD} 11 \mathrm{~b}^{+} \mathrm{CD} 31^{+}$, further indicating that cells devoid of any endothelial marker were extremely rare. Scanning electron microscopy (SEM) verified that the cell membrane of isolated LSECs contained sieve plates with grouped fenestrae, another characteristic feature of the sinusoidal liver endothelium (Figure 1, F and $\mathrm{G})(14,15)$. This established that LSECs isolated by enzymatic liver digestion followed by immunomagnetic selection with mouse endothelium-specific antibody were highly enriched (>97\% ECs) and were thus suitable for our cell transplantation studies.

LSEC transplantation studies in $\mathrm{FVB} / \mathrm{N}$ mice. To assess the fate of transplanted LSECs in the liver, we injected $2 \times 10^{6} \mathrm{FVB} / \mathrm{N}-\mathrm{Tie} 2-$ GFP cells via the portal vein into FVB/N mice of the same genetic background. Recipient animals were subjected to interval analysis for identifying transplanted LSECs in the liver using immunostaining for GFP (Figure 1, H-K). Initially we determined whether donor LSECs would engraft efficiently in the mouse liver after transplantation. Immediately after transplantation, LSECs were distributed in liver sinusoids, and after 3-7 days, transplanted LSECs were integrated in the liver structure and engrafted in the EC layer bounding sinusoids within the liver. Analysis to demonstrate the long-term fate of cells at subsequent periods of 2 weeks and 1,2, and 3 months showed that transplanted LSECs survived in the liver and were distinguishable from other sinusoidal liver cells throughout the duration of our studies (Figure 1, L-S). To measure what fraction of transplanted LSECs engrafted and survived in the long term, we isolated liver cells by enzymatic digestion from mice transplanted with FVB/N-Tie2-GFP LSECs followed by flow cytometry analysis of GFP-expressing cells. This demonstrated that transplanted ECs constituted less than $0.5 \%$ of the total sinusoidal liver EC fraction (see below). As the observed extent of endothelial reconstitution $(<0.5 \%)$ would likely have been less than therapeutic, next we examined whether LSEC engraftment could be enhanced.

To modulate transplanted cell engraftment, we administered an engineered fibronectin-like polymer (FLP) so the principle of receptor-mediated targeting of LSECs to the liver endothelium could be addressed, as demonstrated previously for hepatic targeting of mature hepatocytes (16). Alternatively, we administered a genotoxic pyrrolizidine alkaloid, monocrotaline (MCT), which disrupts the sinusoidal endothelial barrier and facilitates incor- 
A MCT Untreated

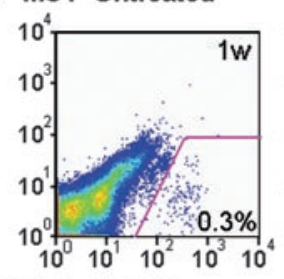

B MCT -Treated
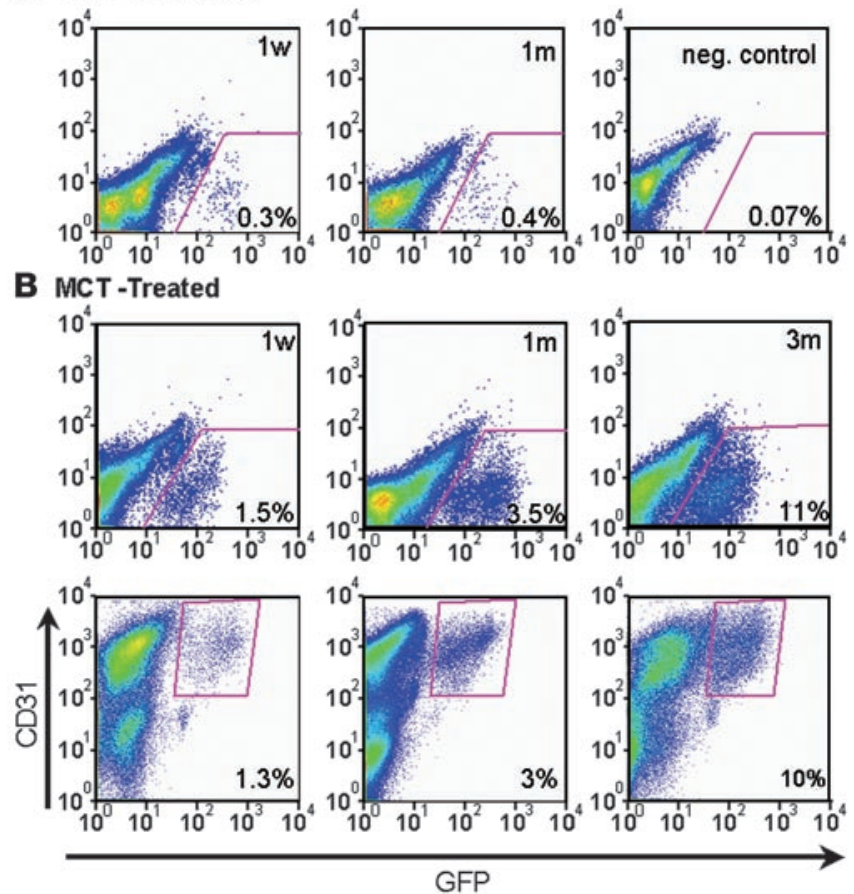

poration of transplanted hepatocytes into the liver parenchyma (17-19). The rationale of this latter approach was based on the fact that MCT is a potent toxin to ECs and causes widespread disruption of endothelia in intact animals $(20,21)$. To blunt potential host immune responses against the GFP reporter in these immunocompetent $\mathrm{FVB} / \mathrm{N}$ mice, our studies incorporated administration of low-dose cyclophosphamide, which in high doses can additionally deplete macrophages and disrupt endothelium (22).

We observed that in FLP-treated mice, intrahepatic retention of transplanted LSECs improved, and there was also moderate improvement in cell engraftment compared with control mice (Figure 1, I and J). On the other hand, LSEC engraftment in MCTtreated mice increased by several-fold compared with untreated controls (Figure $1 \mathrm{~K}$ ). We discovered that after only a single dose of MCT to recipients before cell transplantation, the number of healthy GFP-positive transplanted LSECs rose progressively over 3 months, indicating onset of transplanted cell proliferation (Figure $1, \mathrm{~L}-\mathrm{S})$. This was commensurate with long-lasting perturbations in the liver of LSEC recipient animals that had been exposed to MCT. Although 48 hours after MCT, prior to cell transplantation, serum alanine aminotransferase levels were mildly elevated ( $\sim 3$-fold), indicating acute liver injury, 1 and 2 weeks after cell transplantation, serum alanine aminotransferase levels were normal. Moreover, we did not observe any evidence for hepatic sinusoidal occlusion at the morphological level in animals studied in the short and the long term. The finding of progressive proliferation in transplanted

Table 1

\section{Animal groups}

No conditioning

MCT conditioning

Mean fold change vs. 1 week No conditioning

$P$ values

\section{Figure 2}

Flow cytometric analysis to identify reisolated transplanted FVB/NTie2-GFP cells as a fraction of total nonparenchymal liver cells. (A) Shown are GFP-positive cells in MCT-untreated mice, with transplanted cells constituting less than $0.5 \%$ of NPCs after 1 week and 1 month. Negative control mouse without cell transplantation is shown. (B) Upper panels show GFP-positive cells in MCT-treated mice, in which transplanted cell numbers increased progressively, reaching up to $11 \%$ of NPCs after 3 months. Lower panels show that GFP-positive cells were also CD31 positive.

LSECs suggested that native endothelial liver cells suffering from MCT-induced damage were at a competitive disadvantage compared with transplanted healthy cells (see below).

In previous studies lasting up to 7 days, we established that LSECs transplanted into the liver via portal vein injection survived only in the liver without redistribution to other organs, e.g., lungs, heart, spleen, kidney, or intestine (8). To establish that MCT-induced endothelial injury in additional vascular beds did not promote survival of transplanted LSECs in extrahepatic locations, we surveyed tissues for GFP transgene by PCR. We found that transplanted LSECs were still restricted to the liver in MCTtreated animals (Supplemental Figure 1; supplemental material available online with this article; doi:10.1172/JCI32748DS1). This indicated that studies of the long-term fate of transplanted cells in the liver were appropriate.

Endothelial reconstitution by transplanted FVB/N-Tie2-GFP LSECs. To quantitate the extent of short-term and long-term endothelial reconstitution by transplanted FVB/N-Tie2-GFP LSECs, we isolated liver cells from multiple animals by enzymatic digestion followed by flow cytometric analysis. One week after LSEC transplantation, in mice without MCT pretreatment, transplanted cells constituted $0.3 \% \pm 0.1 \%$ of the nonparenchymal liver cell fraction devoid of hepatocytes (nonparenchymal cells [NPCs]) (Figure 2A). This transplanted cell fraction was constant in mice without MCT over 1 month, indicating absence of transplanted cell proliferation (Figure 2B). However, in mice given MCT before cell transplantation, the number of transplanted LSECs was on average 4-fold greater after 1 week, indicating superior cell engraftment, and transplanted LSECs proliferated to constitute 9\% of NPCs after 3 months (Figure 2, D-F). We demonstrated CD31 expression in GFP-positive transplanted cells to verify their endothelial identity (Figure 2, G-I). Cumulative analysis of these data indicated up to

Cumulative analysis of transplanted LSEC fraction

\section{Time after cell transplantation}

\begin{tabular}{|c|c|c|c|}
\hline 1 week & 1 month & 2 months & 3 months \\
\hline $\begin{array}{c}0.3 \% \pm 0.1 \% \\
(n=6)\end{array}$ & $\begin{array}{c}0.4 \% \pm 0.1 \% \\
(n=3)\end{array}$ & & \\
\hline $\begin{array}{c}1.2 \% \pm 0.1 \% \\
(n=3)\end{array}$ & $\begin{array}{c}3.5 \% \pm 1.2 \% \\
(n=4)\end{array}$ & $\begin{array}{c}4.7 \% \pm 1.2 \% \\
(n=3)\end{array}$ & $\begin{array}{c}9.0 \% \pm 2.0 \% \\
(n=4)\end{array}$ \\
\hline 4 & 11 & 15 & 30 \\
\hline 0.001 & 0.001 & 0.001 & 0.001 \\
\hline
\end{tabular}

Cumulative analysis showing multifold increases in transplanted cell numbers in MCT-treated mice. Data refer to the percentage of reisolated GFP-positive transplanted LSECs in the hepatic NPC fraction, which constituted $100 \%$ of the cells in this analysis. 
A

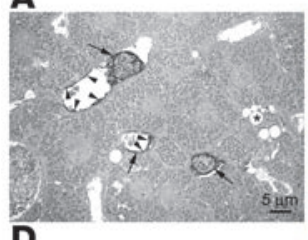

D
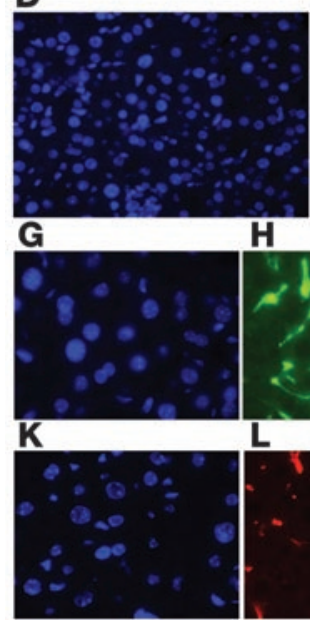

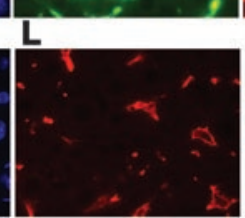

B

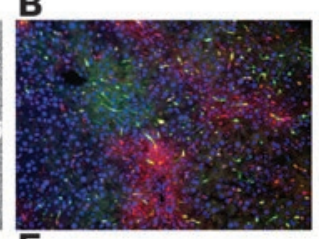

E

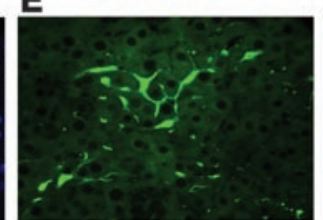

\begin{abstract}
H
\end{abstract}

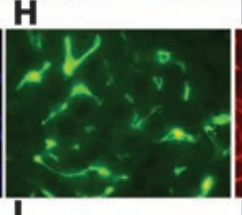

I

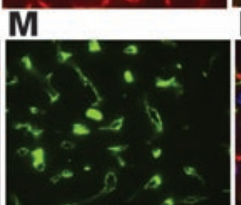

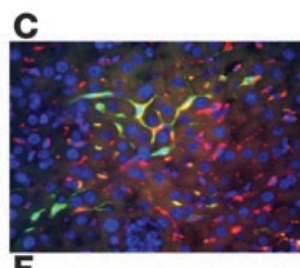

$\mathbf{F}$

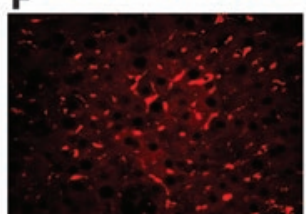

J

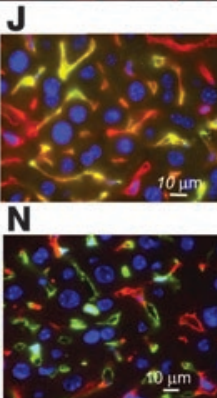

Figure 3

Integrity of transplanted LSECs in the liver. (A) TEM after GFP immunostaining showing integration of transplanted LSECs after 1 week (arrows) with characteristic layering of hepatic sinusoidal endothelium (arrowheads). Asterisk indicates lipid-laden stellate cells in adjacent area. (B-F) Analysis of Dil-Ac-LDL uptake (red) in GFP-positive LSECs (green) producing yellow color in cells containing both. Shown are transplanted GFP-positive LSECs in FVB/N mice containing green, yellow, or red domains due to Dil-Ac-LDL uptake in cells 3 months (E) after cell transplantation. (G-J) Combined staining for GFP $(\mathbf{H})$ and CD31 (I) verifying transplanted cells expressed both markers (yellow). (K-N) Costaining for F4/80 (L, red) and GFP ( $\mathbf{M}$, green) showing absence of F4/80 immunostaining in transplanted LSECs, indicating distinction from Kupffer cells. $(\mathbf{G}$ and $\mathbf{K})$ Nuclei stained with DAPI (blue). Original magnification, $\times 2,700(\mathbf{A})$; $\times 200(\mathbf{B}) ; \times 400(\mathbf{C}-\mathbf{F}) ; \times 630(\mathbf{G}-\mathbf{N})$. Scale bars: $5 \mu \mathrm{m}(\mathbf{A}) ; 10 \mu \mathrm{m}(\mathbf{G}-\mathbf{N})$.

30 -fold increases in transplanted cell numbers over time (Table 1). Tissue analysis by GFP staining showed large numbers of GFPpositive transplanted LSECs in the liver 1 and 3 months after cell transplantation (Figure 1, L-S).

Structural and functional integrity of transplanted LSECs in the liver. Next, we established the structural and functional integrity of reconstituted hepatic endothelium following LSEC transplantation. Transmission electron microscopy (TEM) demonstrated that transplanted LSECs were correctly integrated in the liver endothelium with the space of Disse intervening between LSECs and hepatocytes (Figure 3A). To demonstrate receptor-mediated endocytosis through scavenger receptors in transplanted LSECs, which is a physiological function of ECs, we administered to mice fluorescently labeled 1,1-dioctadecyl-3,3,3,3-tetramethylindocarbocyanine-labeled acetylated LDL (DiI-Ac-LDL) (23). Transplanted LSECs incorporated DiI-Ac-LDL 1, 3, and 7 days after cell transplantation as well as subsequently throughout the 3 -month duration of our studies, including in mice with extensive proliferation of transplanted cells (Figure 3, B-F). This assay established that transplanted LSECs were functionally intact from the beginning. We again verified that transplanted LSECs possessed characteristic endothelial membrane markers, e.g., CD31 (Figure 3, G-J), and were distinct from other cells in liver sinusoids, e.g., Kupffer cells, which display the F4/80 antigen (Figure 3, K-N). Confocal microscopy demonstrated that GFP- and CD31-positive transplanted LSECs were distinct from other sinusoidal cells (Supplemental Videos 1 and 2). This meant that transplanted LSECs physiologically reconstituted the sinusoidal endothelial compartment. Absence of GFP in F4/80-stained Kupffer cells indicated that transplanted cells were not being phagocytosed, implying absence of ongoing transplanted cell destruction.

MCT-induced perturbations in LSECs. Previously, we established that MCT promptly disrupted liver sinusoidal endothelium, which was demonstrable at the morphological level, as well as through impaired clearance of endothelial ligands, e.g., serum hyaluronic acid, within 24-48 hours $(17,18)$. If MCT perturbed the liver in the short term as well as the long term, we should have been able to demonstrate relevant changes in LSECs. For instance, changes in cell signaling molecules regulating endothelial remodeling or angiogenesis should have occurred shortly after MCT treatment. Our studies in animals treated several hours previously with MCT verified such a possibility. We observed prompt increases in the hepatic expression of MMP-9, VEGF receptor-2, and the chemokine receptor CXCR4, specific for stromal cell-derived factor-1 (SDF-1), whereas expression of VEGF or SDF-1 in the liver was unchanged (Figure 4). As MMPs, CXCR4, VEGF, and SDF-1 mediate processes involved in endothelial remodeling during transplanted cell engraftment in the liver $(24,25)$, these findings were in agreement with activation of engraftment-promoting events in MCT-treated animals. In this situation, recruitment of cell-specific receptors would help targeting of transplanted cells to the endothelium whereas matrix-degrading and matrix-reconstituting MMP activities will benefit permanent integration of transplanted cells in the liver structure.

Similarly, MCT should have impaired survival and/or proliferation capacity in native ECs following genotoxicity, which is a known feature of pyrrolizidine alkaloids (20). To analyze changes in survival of native LSECs, we examined FVB/N mice 2-4 weeks after MCT. We chose these times to ensure that acute injury following MCT will have resolved and that ECs will not have been severely perturbed by MCT. The viability of freshly isolated LSECs 2 weeks after MCT was impaired: 74\% $\pm 4 \%$ versus $92 \% \pm 3 \%$ in LSECs from untreated control mice, $P<0.001$. In freshly isolated LSECs from healthy donors, $5 \%$ were undergoing apoptosis as shown by TUNEL, whereas $23 \%$ freshly isolated LSECs from donors 1 month after MCT were positive for TUNEL, $P<0.001$ (Supplemental Figure 2).

Also, we performed studies in MCT-pretreated $\mathrm{FVB} / \mathrm{N}$ recipients with transplantation of LSECs from FVB/N-Tie2-GFP donors treated 2 weeks previously with MCT. These MCT-treated donor cells engrafted in the liver and displayed suitable endothelial markers (Figure 5, A and B). However, the capacity of these cells to proliferate was markedly impaired (Figure 5C). Quantitative analysis of reisolated transplanted cells showed that 1 week after cell transplantation, engraftment of FVB/N-Tie2-GFP LSECs from MCT-treated donors was at least 10 -fold lower than healthy LSEC donors, $0.1 \%$ vs. $1.3 \%$ transplanted LSECs in NPCs by flow cytometry, $P<0.001$ (Figure 5D and Table 2). Similarly, 1 month after cell 


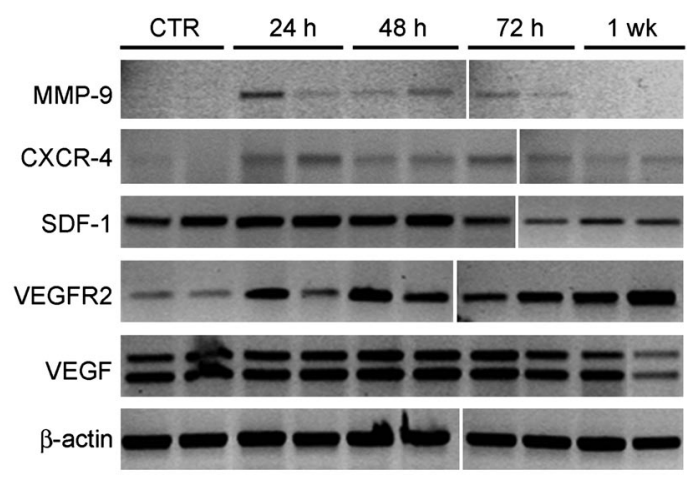

Figure 4

RT-PCR analysis of total liver RNA from FVB/N mice treated with MCT. RT-PCR for several genes (MMP-9, CXCR4, SDF-1, VEGFR2, and VEGF) was performed in mice sacrificed at various times after MCT. For gene expression, liver RNA from 2 mice per condition was analyzed. The data showed increased expression of MMP-9, CXCR4, and VEGFR2.

transplantation, 10-fold fewer transplanted LSECs were recovered from MCT-treated donors compared with healthy donors. In MCTtreated animals in which no cells were transplanted, widespread apoptosis was observed in sinusoidal liver cells, which was different from animals in which transplanted LSECs were undergoing extensive proliferation (Supplemental Figure 3, D-F). To identify cell fractions undergoing cycling, we performed Ki67 staining. In normal mouse liver, ECs did not express Ki67. In contrast, in MCTtreated mice in which transplanted cells were undergoing proliferation, liver sinusoidal cells expressed Ki67 extensively 1 week as well as 1 month after cell transplantation (Supplemental Figure 4, $\mathrm{F}-\mathrm{H})$. Therefore, we concluded that MCT impaired the capacity of native LSECs to survive and to proliferate, thereby offering advantages to transplanted LSECs in the liver.

These mechanisms provided suitable experimental systems for addressing repopulation of the endothelial compartment although potential additional advantages offered by the incorporation of cyclophosphamide required consideration. In FVB/N mice treated with cyclophosphamide alone, transplanted LSECs did not proliferate, indicating that by itself, cyclophosphamide was not responsible for this effect (Figure 2B). Short-term in vitro assays showed that cyclophosphamide did not enhance MCT-

\section{Figure 5}

Perturbations induced by MCT in native LSECs. Analysis of liver from MCT-pretreated FVB/N recipient mice after transplantation of LSECs from FVB/N-Tie2-GFP donor mice that had also been treated with MCT 2 weeks before cells were isolated for transplantation. (A) Tissue sections were immunostained with GFP (green) and F4/80 (red) antibodies 1 week after cell transplantation. (B) Higher magnification view of GFP and CD31 staining in a section from mouse 1 week after cell transplantation. Arrows indicate GFP-positive transplanted LSECs displaying CD31. (C) Immunostaining to identify transplanted LSECs with GFP (green) along with CD31 endothelial marker (red) 1 month after cell transplantation. (D) FACS analysis of MCT-treated FVB/N-Tie2-GFP transplanted LSECs recovered 1 week and 1 month after transplantation. The identities of CD31+/GFP LSECs for typical endothelial markers, endoglin and Flk-1, and the myeloid marker CD11b are shown in the last 6 plots of LSECs isolated from mice 1 week after cell transplantation. Nuclei stained with DAPI (blue). Original magnification, $\times 200(\mathbf{A}, \mathbf{C}) ; \times 630$ (B). Scale bar: $10 \mu \mathrm{m}(\mathbf{B})$. induced cytotoxicity in LSECs (Supplemental Figure 5). However, as cyclophosphamide may not be toxic to cultured LSECs without hepatocytes (26), we could not exclude the possibility of different in vivo effects.

Function of transplanted bealthy LSECs in NOD/SCID bemophilia A mice. As plasma FVIII activity is undetectable in hemophilia A mice, we hypothesized that transplanted LSECs producing and releasing sufficient amounts of FVIII in blood would result in therapeutic correction of hemophilia A. These FVIII knockout mice constitute an excellent model for addressing cell therapy in hemophilia A (27-29). We transplanted healthy FVIII-expressing LSECs from FVB/N-Tie2-GFP donors via the portal vein into hemophilia A mice tat were backcrossed 10 times with $\mathrm{T}$ and $\mathrm{B}$ cell-deficient NOD.CB17-Prkdcscid/J mice. This was done to eliminate the possibility of immune responses against FVIII secreted by transplanted cells as well as against transplanted cells themselves. We administered MCT to hemophilia A mice $(n=31)$ from 1 to 2 days before cell transplantation. To perform surgery in these mice, we administered 4 units of recombinant human FVIII once, which exhibits terminal half-life of 4.1 hours in mice (30).

Two mice died before reaching the 1-month time point and were lost to analysis. Subsequently, in 1 set of studies, we examined tissues and plasma from 16 animals 1 month after LSEC transplantation, although plasma from 1 of these animals was lost during sampling. RT-PCR demonstrated FVIII mRNA in the liver of virtually all mice (Figure 6A). Again, we identified transplanted LSECs with GFP and CD31, distinct from F4/80-reactive Kupffer cells (Figure 6, B and C, and Figure 3, G-N). The relative abundance of hepatic FVIII mRNAs correlated with the prevalence of transplanted LSECs in the liver of hemophilic mice. We demonstrated plasma FVIII activity with a sensitive fluorogenic thrombin generation test (31), arbitrarily using a stringent cutoff of more than $10 \%$ plasma FVIII activity to define therapeutic correction. Significant correlation was found between the overall extent of liver repopu-
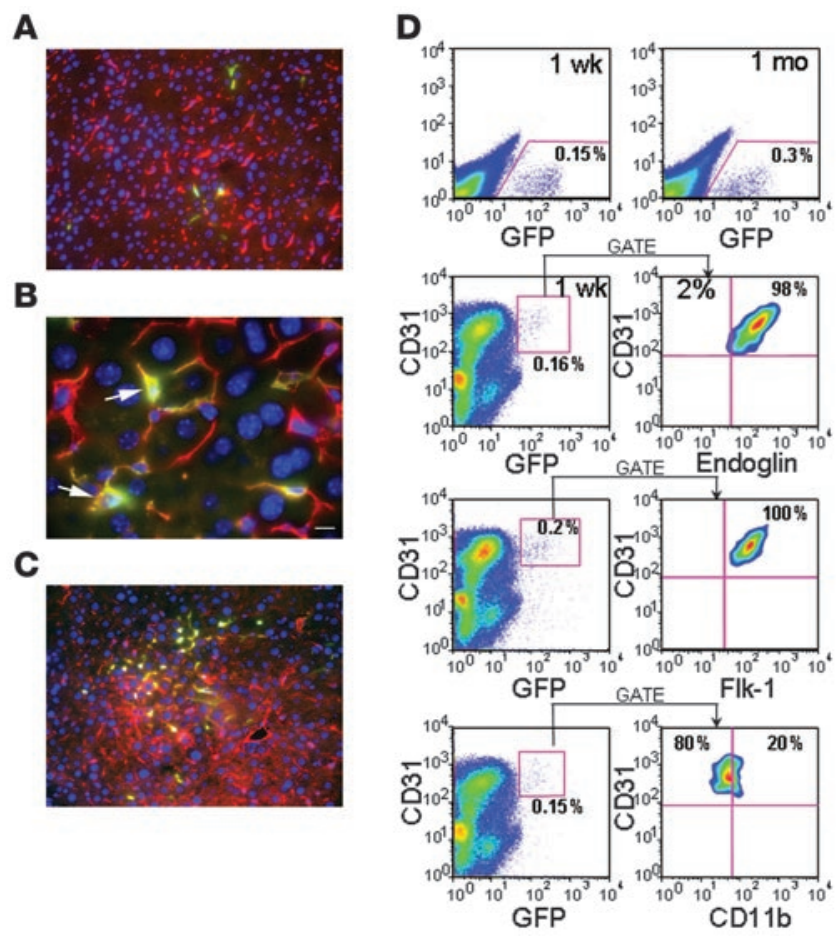
Table 2

Recovery of transplanted cells

\section{Experimental condition}

GFP+ fraction in NPCs

\section{One week after cell transplantation}

Mouse 1

Mouse 2

$0.15 \%$

Mouse 3

$0.13 \%$

$0.05 \%$

One month after cell transplantation

Mouse 1

Mouse 2

$0.29 \%$

$0.27 \%$

Shown are percentages of hepatic NPCs of GFP+ LSECs recovered from MCT-treated FVB/N mice injected with MCT-treated FVB/N Tie2GFP LSECs 1 week and 1 month after cell transplantation.

lation with transplanted LSECs and plasma FVIII activity. Our criterion of therapeutic correction indicated that transplantation of healthy LSECs corrected hemophilia A in 13 of 15 mice (87\%). Mean FVIII activity in these mice was $19 \% \pm 4 \%$ of normal plasma activity (range: 14\%-25\%) (Figure 6D). In 2 mice judged to be without therapeutic correction, FVIII activity was either undetectable or only $7 \%$. Transplanted cells were less prevalent in the liver of these 2 mice compared with mice showing therapeutic correction.

In hemophilia A mice subjected to LSEC transplantation, FVIII antigen appeared in the blood (Figure 7A), corresponding to increased plasma FVIII activity. We did not detect antibodies against FVIII in hemophilia A mice treated with healthy LSECs. As NOD/SCID mice lack the intrinsic ability to mount antibody responses due to lack of B (and T) cells, this was expected. To verify that plasma FVIII levels increased progressively in hemophilia A mice during proliferation of transplanted LSECs, we obtained blood samples serially in another set of mice over up to 2 months (Figure 7B). Plasma FVIII levels increased over time in relationship with increased liver repopulation with transplanted cells. This analysis showed that plasma FVIII activity increased from $19 \% \pm 11 \%$ after 2 weeks to $36 \% \pm 16 \%$ after 2 months following cell transplantation, although the difference did not achieve statistical significance due to animal-to-animal variabilities. Nonetheless, plasma FVIII activity was not lost in any of the hemophilia A mice, which indicated persistent function of transplanted cells.

\section{Figure 6}

Therapeutic efficacy of transplanted LSECs in NOD/SCID hemophilia A mice. (A) RT-PCR analysis of total liver RNA showing FVIII mRNA in virtually all treated mice (lanes 2-13). The samples were analyzed for $\beta$-actin in parallel to verify RNA integrity. Lane 1, negative control with PCR mix alone; lane 14, healthy mouse liver RNA as positive control. (B) Immunostaining of liver showing transplanted FVB/N-Tie2GFP LSECs in NOD/SCID hemophilia A mouse (green) with coexpression of CD31 endothelial marker (red in native cells; yellow in transplanted cells). (C) GFP-positive transplanted cells (green) distinct from F4/80-expressing Kupffer cells (red). (D) Plasma FVIII activity in NOD/SCID hemophilia A mice 1 month after transplantation of FVB/N-Tie2-GFP LSECs with therapeutic correction requiring more than $10 \%$ plasma FVIII. Original magnification, $\times 200$ (B and C).

A

Restoration of plasma FVIII activity should have corrected the bleeding phenotype in hemophilia A mice. This was examined in another set of studies. In hemophilia A mice 2 months following LSEC transplantation, tail cut-induced bleeding stopped after 15-120 minutes, and 6 of 6 hemophilia A mice survived (100\%). In contrast, in untreated control hemophilia A mice, bleeding continued and proved fatal.

We expected that in hemophilia A mice, other coagulation factors and cofactors would be expressed normally, e.g., vWF or coagulation factor IX (FIX), since no other gene was disrupted. This was demonstrated by the presence of vWF and FIX mRNAs (Figure 8A) as well as vWF protein in LSECs from hemophilia A mice (Figure 8, $B$ and C, and Supplemental Video 3 showing confocal microscopy). Similarly, hemophilia A mice subjected to LSEC transplantation also expressed vWF (Figure 8D). It was difficult to discern whether restoration of FVIII expression in these animals had counterregulatory effects on vWF expression. Finally, to determine whether ECs in other organs could offer opportunities to reconstitute FVIII deficiency, we studied FVIII expression in several organs (Figure $8 \mathrm{E})$. FVIII mRNA was present in spleen, lungs, and bone marrow, although at lower levels than in liver. To entertain the possibility of cell transplantation studies, we isolated ECs from the mouse spleen by immunomagnetic cell sorting. However, the yield of splenic ECs was extremely limited, and we could isolate less than $5 \times 10^{5} \mathrm{ECs}$ from the spleen, which restricted in vivo studies.

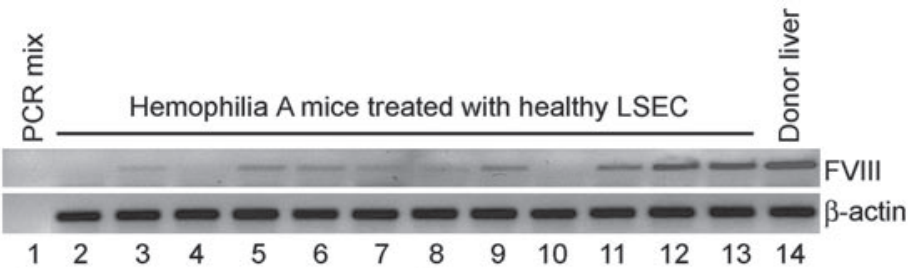

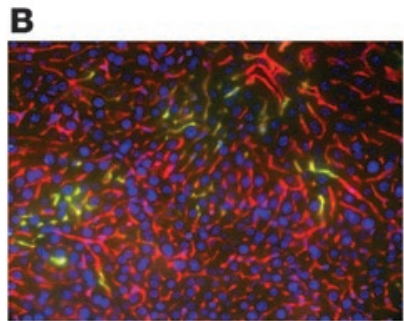

CD31 GFP DAPI

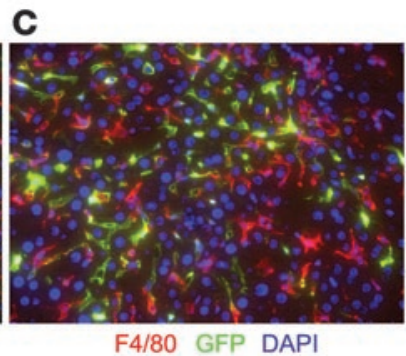

D

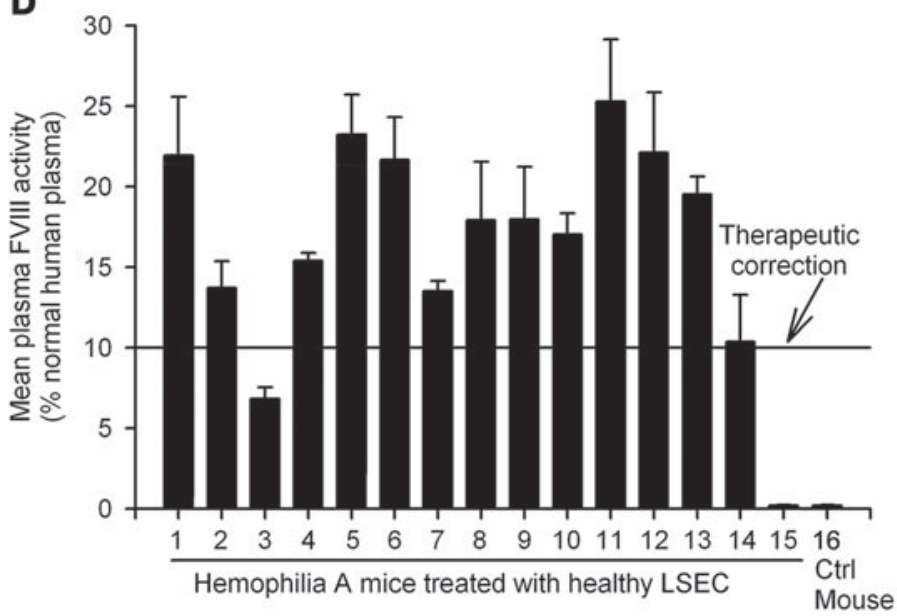


A

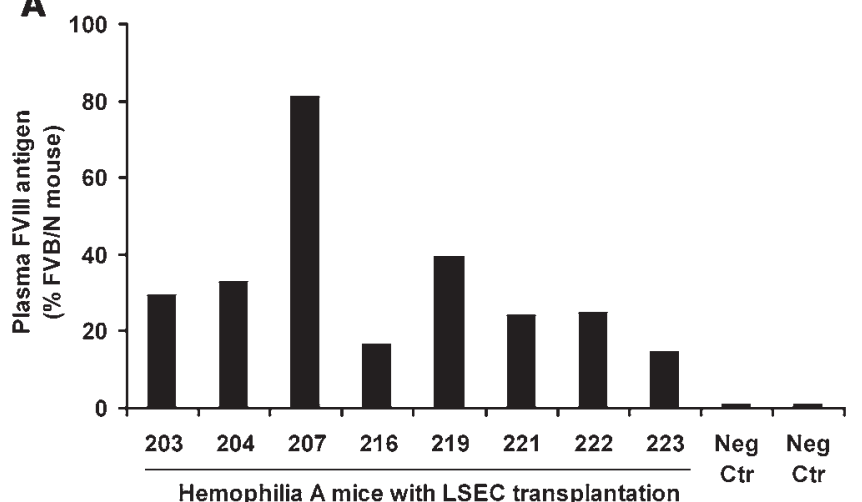

B

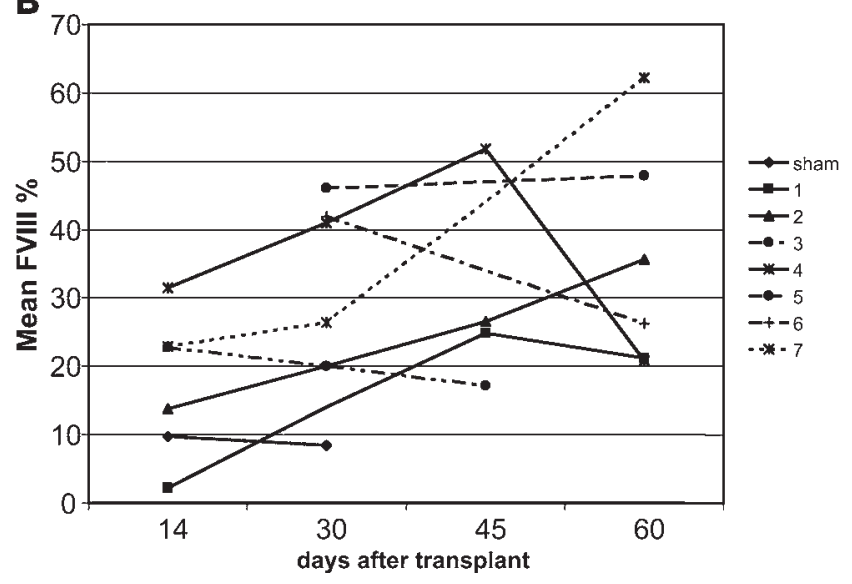

\section{Discussion}

Although mature ECs reconstituted hepatic sinusoidal endothelium in our studies, additional cell types, including circulating mononuclear cell-derived or other endothelial progenitor cells, may home to areas of endothelial damage and contribute to vasculogenesis or neoangiogenesis (32-35) and may improve outcomes in animals with liver disease (36). In our studies, donor cells were mature LSECs, and contamination with other cell types was essentially of no significance. Under suitable growth factor stimulation conditions, primary mature LSECs can proliferate in vitro, and as now shown in our studies, also in vivo. Judging from our experience in isolating ECs from the spleen, organs other than the liver may not necessarily provide more ECs. Although isolation of primary cells from human livers requires significant expertise, donor livers are processed routinely to isolate hepatocytes and other liver cells. We considered that transplantation of $2 \times 10^{6} \mathrm{LSECs}$ in mice represented $10 \%$ of the endothelial compartment and $5 \%$ of the NPC fraction, assuming that LSECs, NPCs, and hepatocytes constituted $20 \%, 40 \%$, and $60 \%$, respectively, of $1 \times 10^{8}$ total liver cells. In principle, a single donor human liver should generate sufficient numbers of LSECs to treat multiple patients if only $10 \%$ of the LSECs were needed for transplantation.

Reisolation of up to $0.5 \%$ transplanted LSECs in NPCs from mice without MCT preconditioning suggested that only $10 \%$ of the transplanted LSECs engrafted, which was generally similar to the engraftment efficiency of primary hepatocytes in the liver (37). On the other hand, pretreatment of mice with FLP and MCT, especially MCT, significantly enhanced LSEC engraftment, also resembling the effect of MCT on the engraftment of transplanted hepatocytes, which too is facilitated by the disruption of the

\section{Figure 7}

FVIII antigen in LSEC-transplanted hemophilia A mice and serial analysis of FVIII activity in cell recipients. (A) Hemophilia A mice 1 month after transplantation of FVB/N-Tie2-GFP LSECs with therapeutic correction. Neg Ctr, untreated hemophilia $A$ mice. Each bar represents FVIII antigen level from an individual mouse after cell transplantation. (B) Plasma FVIII activity in hemophilia A mice after cell transplantation showing serial bleeding from 7 mice starting 2 weeks after surgery over a period of 60 days with therapeutic correction requiring more than $10 \%$ plasma FVIII. Mouse indicated as sham received vehicle alone.

sinusoidal endothelial barrier (17-19). The mechanism by which FLP works is quite different because MCT damages cells, whereas FLP engages extracellular matrix receptors without causing cell damage (16). Subsequently, transplanted LSECs proliferated in MCT-pretreated mice due to impairment in the survival and proliferation capacity of native LSECs, as shown by our studies, which were in agreement with the propensity of MCT to induce persistent cell-cycle arrest, such as through G2/M block, in ECs (38, 39). Consequently, the extent of liver repopulation with LSECs reached $10 \%-20 \%$ of the endothelial mass, judging from the analysis of cell fractions in our studies. In contrast, MCT did not affect hepatocytes in this manner, unless hepatic injury was redirected by additional drug toxicities or accumulation of toxic MCT intermediates through activation of cytochrome P450-induced metabolism of MCT in hepatocytes (18). The principle of selective injury in native cells can possibly be applied to repopulation of EC compartments in other vascular beds and assessment the potential of cell-cell interactions in various conditions.

To consider clinical applications, substitutes for MCT will be necessary, since this substance has oncogenic potential. As cyclophosphamide and doxorubicin, which are already in clinical use, were also effective for disrupting the hepatic endothelial barrier to improve engraftment of transplanted hepatocytes $(22,40)$, potential alternative approaches should be feasible. Identification of suitable changes in the liver that promote proliferation of transplanted LSECs should provide impetus for further research into suitable drugs capable of reproducing these mechanisms.

It was particularly noteworthy that transplanted LSECs maintained physiological function in the liver. Our studies unequivocally established that LSECs are the major source of FVIII. For targeted cell and gene therapy in hemophilia $\mathrm{A}$, identification of the cell type(s) capable of producing FVIII will be of enormous value. Although whole liver transplantation in people with hemophilia A promptly resulted in the restoration of plasma FVIII activity, supporting a role of the liver in FVIII synthesis (41), the specific cell type(s) responsible for producing FVIII had remained elusive because FVIII mRNA was expressed in hepatocytes, LSECs, and macrophages $(2,12,29)$. The cell type-specific origin of FVIII was not resolved by further studies since transplantation of healthy LSECs (29), fetal spleen containing vascular endothelium (28), and hepatocytes (42) rescued hemophilia A mice. FVIII mRNA is particularly well expressed in hepatocytes and LSECs, although quantitative assays demonstrated greater mRNA expression in the latter (2). We consider that LSECs are the major source of FVIII protein, since LSEC transplantation in hemophilia A mice restored plasma FVIII activity levels to $14 \%-25 \%$ of normal, which correlated to the extent by which the hepatic endothelium was replaced by transplanted LSECs. It should be noteworthy that these levels of plasma FVIII activity eliminate spontaneous bleeding in hemophilia A. 
A

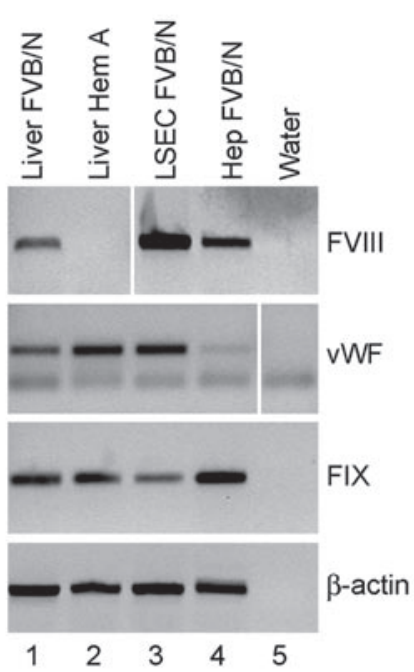

D

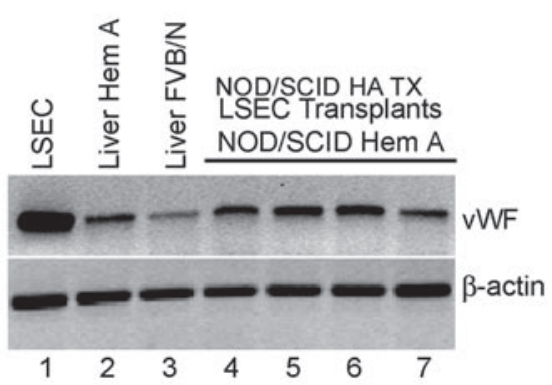

B
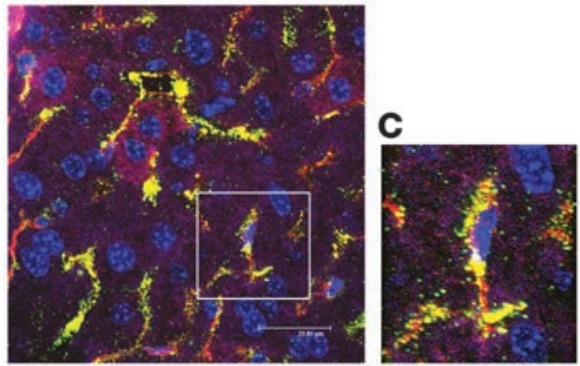

E

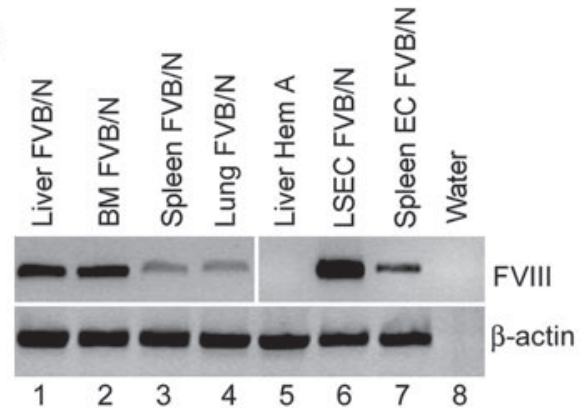

Figure 8

Expression of coagulation factors in ECs. (A) RT-PCR of total liver RNA for FVIII, FIX, and VWF. Lane 1, liver from healthy FVB/N mouse; lane 2, liver from hemophilia A mouse; lane 3, LSECs from healthy FVB/N mouse; lane 4, hepatocytes from healthy FVB/N mouse; and lane 5, PCR mix alone. (B and C) Immunostaining of NOD/SCID hemophilia A mouse liver showing coexpression of CD31 endothelial marker (red) and vWF (green) in liver sinusoids; cells expressing both appear yellow. $\mathbf{C}$ shows enlargement of the boxed area in B. Nuclei were stained with DAPI (blue). In B, confocal microscopy demonstrates vWF expression in LSECs costained for CD31. Scale bar: $24 \mu \mathrm{m}$ (B). Original magnification, $\times 630$ (C). (D) RT-PCR of total liver RNA showing VWF mRNA expression. Lane 1, FVB/N donor LSECs; lane 2, hemophilia A mouse liver; lane 3, FVB/N mouse liver; lanes 4-7, liver of hemophilic mice after LSEC transplantation. (E) RT-PCR of total RNA for FVIII expression. Lanes 1-4, FVB/N mouse liver, bone marrow, spleen, and lung, respectively; lane 5, hemophilia A mouse liver; lane 6, FVB/N LSECs; lane 7, FVB/N ECs from spleen; and lane 8, mix alone. Expression of $\beta$-actin verified RNA integrity in $\mathbf{A}, \mathbf{D}$, and $\mathbf{E}$.
No other major cell type capable of producing FVIII had been transplanted in our studies. While plasma FVIII may originate from additional sources, e.g., peripheral mononuclear cell derivedblood outgrowth ECs (11) or lung microvascular ECs (43), LSECs possess the intrinsic advantage of simultaneously synthesizing, sequestering, and releasing $\mathrm{VWF}$, which stabilizes and protects FVIII against premature degradation or cellular incorporation (44). Moreover, LSECs play roles in immunoregulation, which could potentially be harnessed to avoid deleterious immune responses against FVIII (3). Therefore, the ability of transplanted healthy LSECs to treat FVIII deficiency in hemophilia A should offer exciting new therapeutic approaches.

Development of clinical applications with transplanted ECs for vascular reconstitution should be facilitated by the identification of effective sources of donor cells. Since the supply of donor human organs is limited, whether donor cells could be derived from alternative sources, including stem cells, will need consideration. The possibility of using genetically modified autologous stem cells after appropriate expansion in vitro could help avoid allograft-related issues.

\section{Methods}

Animals and procedures. The Animal Care and Use Committee of the Albert Einstein College of Medicine approved animal use in conformity with the National Research Council's Guide for the care and use of laboratory animals (United States Public Health Service publication. Revised 1996).

Mice of 7-10 weeks of age were used. TgN(TIE2GFP)287Sato/J transgenic mice in the FVB/NJ background (Jackson Laboratory) served as cell donors. After initial studies to develop cell isolation procedures, $20 \mathrm{TgN}$ (TIE2GFP)287Sato/J mice were used for cell transplantation studies with FVB/N mice (National Cancer Institute) serving as syngeneic cell recipients ( $n=22$ for final studies). Hemophilia A mice were originally produced by disruption of the FVIII gene through insertion of a neomycin gene cassette in the $3^{\prime}$ end of exon 16 (27). To generate NOD/SCID hemophilia A mice, we backcrossed over 10 generations of female hemophilia $\mathrm{A}$ mice in 129-C57BL/6J background with male NOD.CB17-Prkdcscid/J mice (Jackson Laboratory). We verified the genotype of hemophilia knockout mice by PCR as described (27). For demonstrating therapeutic correction, we used $32 \mathrm{NOD} / \mathrm{SCID}$ hemophilia A mice as recipients of healthy LSECs from $\operatorname{TgN}(\mathrm{TIE} 2 \mathrm{GFP}) 287 \mathrm{Sato} / \mathrm{J}$ transgenic mice $(n=20)$. Prior to cell transplantation surgery in NOD/SCID hemophilia A mice, we administered 4 U of FVVIII protein by tail-vein injection (Refacto; Wyeth Research). Tailclip challenge was used to assess correction of the hemophilia phenotype as previously described (45). Mice were anesthetized with $5 \%$ isoflurane and maintained on $1.5 \%$ isoflurane anesthesia during surgery. The portal vein was exposed by laparotomy, and $2 \times 10^{6}$ LSECs were injected in $0.3 \mathrm{ml}$ serum-free DMEM (GIBCO; Invitrogen) using 27-gauge needles. Hemostasis was secured by brief pressure for up to 5 minutes on injection sites. Some animals received $53 \mathrm{mg} / \mathrm{kg}$ FLP (Deepwater Chemicals Inc.) in saline intraportally immediately before cell transplantation. Other animals received $200 \mathrm{mg} / \mathrm{kg}$ MCT (Sigma-Aldrich) in saline i.p. 24-72 hours before intraportal cell transplantation or 2-4 weeks before cell isolation for analyzing the effects of MCT on donor LSECs. To blunt immune responses against GFP transgene reporter, FVB/ $\mathrm{N}$ mice were given $30-50 \mathrm{mg} / \mathrm{kg}$ cyclophosphamide (CYTOXAN; Bristol-Myers Squibb) in normal saline i.p. twice per week for the duration of the study starting from the day of surgery (46). 
Cell isolation and characterization. Liver was perfused at $5 \mathrm{ml} / \mathrm{min}$ via portal vein for 15 minutes with buffer at $37^{\circ} \mathrm{C}$ containing $1.9 \mathrm{mg} / \mathrm{ml}$ EGTA, for 2 minutes with buffer lacking EGTA, and for 7-9 minutes with buffer containing $0.03 \%(\mathrm{w} / \mathrm{v})$ collagenase and $5 \mathrm{mM} \mathrm{CaCl}_{2} \cdot 2 \mathrm{H}_{2} \mathrm{O}$. The perfusion buffer contained $10 \mathrm{mmol} / \mathrm{l} \mathrm{HEPES}, 3 \mathrm{mmol} / \mathrm{l} \mathrm{KCl}, 130 \mathrm{mmol} / \mathrm{l} \mathrm{NaCl}$, $1 \mathrm{mmol} / 1 \mathrm{NaH}_{2} \mathrm{PO}_{4} \cdot \mathrm{H}_{2} \mathrm{O}$, and $10 \mathrm{mmol} / \mathrm{l}$ D-glucose, $\mathrm{pH} 7.4$ (Sigma-Aldrich; collagenase from Worthington Biochemical Corp.). The liver was dissociated in perfusion buffer, and cells were passed through Dacron fabric with $80-\mu \mathrm{m}$ pores and centrifuged twice under $50 \mathrm{~g}$ for 5 minutes to remove hepatocytes. NPCs in the supernatant were washed and pelleted under $350 \mathrm{~g}$ for 10 minutes and fractionated with Percoll (Sigma-Aldrich) in initial experiments as described (8). Additionally, after lysing red blood cells in $155 \mathrm{mM} \mathrm{NH}_{4} \mathrm{Cl}, 10 \mathrm{mM} \mathrm{KHCO}_{3}$, and $0.1 \mathrm{mM}$ EDTA for 6 minutes on ice, LSECs were selected from NPC fraction by immunomagnetic sorting (Miltenyi Biotec Inc.). For this, $2 \times 10^{7}$ cells were resuspended in $200 \mu \mathrm{l}$ separation buffer (PBS, pH 7.2, 2 mM EDTA, 0.5\% BSA; Sigma-Aldrich) along with $10 \mu \mathrm{l} / 1 \times 10^{7}$ cells of anti-CD45-conjugated magnetic beads (Miltenyi Biotec) for 20 minutes at $4^{\circ} \mathrm{C}$. Cells were pelleted under $350 \mathrm{~g}$ for $8 \mathrm{~min}$ utes at $4^{\circ} \mathrm{C}$, resuspended in $500 \mu \mathrm{l}$ separation buffer, and applied to MS Separation Columns (Miltenyi Biotec), and CD45-negative cells were collected for a round of positive selection using anti-LSEC-conjugated magnetic particles (Miltenyi Biotec) for 30 minutes at $4^{\circ} \mathrm{C}$. Isolated LSECs were resuspended in serum-free DMEM and pelleted under $350 \mathrm{~g}$ for 8 minutes at $4^{\circ} \mathrm{C}$ before transplantation. Cell viability was determined with trypan blue dye exclusion. The efficiency of cell separation was verified by staining cells with allophycocyanin-labeled anti-mouse CD31 ( $1 \mu \mathrm{l} / 10^{6}$ cells; BD Biosciences - Pharmingen) followed by analysis using FACSCalibur flow cytometer equipped with CellQuest software (BD Biosciences).

Cell viability assay using thiazolyl blue dye. Immunomagnetically sorted LSECs were plated at $4 \times 10^{5} / \mathrm{cm}^{2}$ and cultured overnight in collagen-coated dishes with 199 Medium (Sigma-Aldrich) containing 20\% FBS, $100 \mu \mathrm{g} / \mathrm{ml} \mathrm{EC}$ growth factor (Sigma-Aldrich), and antibiotics (GIBCO; Invitrogen). After 1 day, thiazolyl blue dye (MTT) was added to a final concentration of $1 \mathrm{mg} / \mathrm{ml}$ for 2 hours at $37^{\circ} \mathrm{C}$. Cells were solubilized in DMSO, and the reaction product was measured at $580 \mathrm{~nm}$ with background subtraction at $675 \mathrm{~nm}$.

SEM. To demonstrate fenestrae in LSECs, SEM was performed. Immunomagnetically sorted LSECs were maintained overnight on glass coverslips in collagen-coated dishes. Cultured cells were fixed in $1 \%$ osmium tetroxide, $0.1 \mathrm{M}$ sodium cacodylate, $0.2 \mathrm{M}$ sucrose, $5 \mathrm{mM} \mathrm{MgCl}_{2}$, $\mathrm{pH} 7.4$, (SEM buffer) for 5 seconds, followed by 2 changes of $2.5 \%$ glutaraldehyde in SEM buffer. Cells were postfixed in $1 \%$ osmium tetroxide in SEM buffer, dehydrated through graded ethanol, critical point dried using liquid carbon dioxide in Tousimis Samdri 795 Critical Point Dryer, and sputter coated with gold palladium in Denton Vacuum Desk-2 Sputter Coater. Cells were examined with a JEOL JSM6400 microscope (JEOL USA Inc.) under $10 \mathrm{kV}$ accelerating voltage.

Tissue analysis. For immunofluorescence studies, liver samples were fixed in $4 \%$ paraformaldehyde in PBS, pH 7.4, (PBS - PAF), equilibrated in $30 \%$ sucrose, embedded in optimum cooling temperature resin, and frozen in 2 -methylbutane at $-80^{\circ} \mathrm{C}$. Cryostat sections of 5- to 6 - $\mu \mathrm{m}$ thickness were blocked in buffer containing $5 \%$ goat serum, $1 \%$ BSA, and $0.1 \%$ Triton X-100 in PBS and incubated with rabbit anti-GFP (1:300; Molecular Probes) alone and with rat anti-mouse F4/80 (1:500; Serotec) or rat anti-mouse CD31 antibody (1:100; BD Biosciences - Pharmingen). For vWF staining, tissue sections were stained with rabbit anti-vWF antibody (1:100; Sigma-Aldrich). For Ki67 staining, tissue sections were fixed in $1 \%$ PAF, postfixed with ethanol-acetic acid (2:1), and stained with rabbit anti-Ki67 antibody (1:1000; Vector Laboratories Inc.). Sections were incubated with Alexa Fluor 488- or Alexa Fluor 546-conjugated goat anti-rabbit IgG and with Alexa Fluor 488- or Alexa Fluor 546-conjugated goat anti-rat IgG using DAPI-Antifade for nuclear staining (Molecular Probes). To demonstrate lipoprotein uptake in transplanted LSECs in vivo, $50 \mu \mathrm{l}$ DiI-Ac-LDL (Biomedical Technologies) was injected i.v. via tail vein into mice (23). After 2 hours, animals were euthanized; liver samples were fixed as described above. $6-\mu \mathrm{m}$-thick liver cryosections were prepared followed by counterstaining of nuclei with DAPI. Tissue sections were examined under epifluorescence, and images were acquired with an Observer.Z1 fluorescence microscope (Carl Zeiss Microimaging Inc.). Microscopy was performed in some instances on the same tissue sections with Leica AOBS Laser Scanning Confocal Microscope, and rotational images and $\mathrm{z}$ section images were acquired.

Analysis of apoptosis. TUNEL assay was performed with a commercial kit (Apoptag; Serologicals Corp.) as described by the manufacturer. Isolated LSECs were either cytospun onto glass slides or were cultured as described above for 24 hours. Frozen tissues were used to obtain cryosections that were fixed with PAF and postfixed with ethanol-acetic acid (2:1). Nuclei were counterstained with DAPI-Antifade (Molecular Probes).

$T E M$. To establish the ultrastructural integrity of transplanted cells, TEM was performed. Liver from transplanted cell recipients and untreated control mice was removed, and 1- to 2-mm-thick tissue slices were placed in icecold $4 \%$ paraformaldehyde in $0.1 \mathrm{M}$ phosphate buffer, $\mathrm{pH} 7.4$, for 3 hours with continuous shaking, after which slices were rinsed in cold phosphate buffer. Tissue sections of approximately $15-\mu \mathrm{m}$ thickness were made with Lancer Vibratome (Polysciences) and exposed sequentially to primary rabbit anti-GFP (1:200; Molecular Probes) and secondary donkey antirabbit-horseradish peroxidase IgG (1:1000; Jackson ImmunoResearch), followed by incubation with 3,3'-diaminobenzidine tetrahydrochloride (DAB) (Sigma-Aldrich) at $\mathrm{pH} 7.0$. These tissue sections were postfixed in $1 \%$ osmium tetraoxide and embedded in epon (Polysciences). Ultrathin 50-nm sections were prepared with LKB ultramicrotome, stained with lead citrate, and examined under a Philips 300 electron microscope (FEI Co.) (47).

Flow cytometric analysis. To quantitate the extent of liver repopulation with GFP-positive cells, we performed flow cytometric analysis. Liver cells were isolated from mice subjected to cell transplantation by collagenase digestion of liver, and hepatocytes were removed under $50 \mathrm{~g}$ as described above. From the total nonparenchymal liver cell fraction, $2 \times 10^{6}$ cells were incubated with $3 \mu \mathrm{l}$ anti-mouse CD16/CD32 ( $1 \mu \mathrm{g} / 10^{6}$ cells; Fc $\gamma$ III/II receptor, $\mathrm{BD}$ Biosciences - Pharmingen) on ice. After 15 minutes, allophycocyanin-labeled anti-mouse CD31 or NK.1 $\left(1 \mu \mathrm{l} / 10^{6}\right.$ cells; BD Biosciences - Pharmingen) was added and cells were kept for 30 minutes on ice and/or with PE-conjugated anti-mouse CD11b, CD45 ( $1 \mu \mathrm{l} / 10^{6}$ cells; BD Biosciences Pharmingen), endoglin (5 $\mu \mathrm{l} / 10^{6}$ cells; R\&D), Tie2, or Flk-1 ( $2 \mu \mathrm{g} / 10^{6}$ cells; eBioscience). Cells were washed and resuspended in $4 \% \mathrm{FBS} / \mathrm{PBS}$. To identify nonviable cells, $1 \mu \mathrm{g} / \mathrm{ml}$ propidium iodide (Sigma-Aldrich) was added. Flow cytometry was performed using FACSCalibur with $1 \times 10^{6}$ events per sample. Data were analyzed with CellQuest software (BD Biosciences - Pharmingen) or Flowjo FACS software (Tree Star Inc.).

RT-PCR assays. Total RNA was extracted by Trizol Reagent (Invitrogen) and treated with DNase (RNase-free DNase; QIAGEN). cDNA was prepared from 2 to $4 \mu \mathrm{g}$ total RNA using the Omniscript RT Kit (QIAGEN).

For FVIII, FIX, and vWF, equal amounts of cDNAs were subjected to PCR with Hot StartTaq DNA Polymerase (QIAGEN) using $95^{\circ} \mathrm{C}$ for initial denaturation followed by 35 cycles of denaturation at $94^{\circ} \mathrm{C}$ for 30 seconds, annealing at $50^{\circ} \mathrm{C}$ for 45 seconds (for FVIII), or $60^{\circ} \mathrm{C}$ for 45 seconds (for FIX) and $62^{\circ} \mathrm{C}$ for 45 seconds (vWF), extension at $72^{\circ} \mathrm{C}$ for 1 minute, and final extension at $72^{\circ} \mathrm{C}$ for 7 minutes. FVIII primers were $5^{\prime}$-CCCTATTATAAGAGCAGAAGTTGA-3' (forward) and 5'-CCAATTAATCCCGAGTGCATATC-3' (reverse), as previously described (48).

FIX primers were 5'-CTCGAGTTGTTGGTGGAGAAAACG-3' (forward) and $5^{\prime}$-TTTTCCCCAGCCACTGACATAGCCA-3' (reverse), as described (2). vWF primers were $5^{\prime}$-TGTTCATCAAATGGTGGGCAGC-3' (forward) and 5'-ACAGACGCCATCTCCAGATTCA-3' (reverse), as described (49). 
For other genes, cDNAs were amplified with Platinum PCR Supermix (Invitrogen) as follows: 30 cycles at $94^{\circ} \mathrm{C}$ for 3 minutes, $94^{\circ} \mathrm{C}$ for 30 seconds, $55^{\circ} \mathrm{C}$ or $57^{\circ} \mathrm{C}$ for 1 minute, $72^{\circ} \mathrm{C}$ for 1 minute, and final elongation at $72^{\circ} \mathrm{C}$ for 7 minutes. The primers were as follows: VEGF: 5'-TCTTCAAGCCGTCCTGTGTG-3' (forward) and 5'-AGGAACATTTACACGTCTGC-3' (reverse); VEGFR2: 5'-CGAGTCTGTCTACCTTGGAGGC-3' (forward) and 5'-CAGCCTGAGCCTTTACCGC-3' (50); MMP-9: $5^{\prime}$-TTGAGTCCGGCAGACAATCC-3' (forward) and 5'-CCTTATCCACGCGAATGACG-3' (reverse) (51); CXCR4: 5'-AGCCTGTGGATGGTGGTGTTTC-3' (forward) and 5'-CCTTGCTTGATGACTCCCAAAAG-3' (reverse) (52); SDF-1 $\alpha$ : 5'-CCAAGGTCGTCGCCGTGCTG-3' (forward) and $5^{\prime}$-CTCCAGGTACTCTTGGATCCAC-3' (reverse) (53). SDF-1 primers were designed using GenBank NM_001012477 sequence. Mouse $\beta$-actin primers were 5'-GTGGGGCGCCCCAGGCACCA-3' (forward) and 5'CTTCCTTATTGTCACGCACGATTTC-3' (reverse) (52).

PCR products were resolved in $1.5 \%$ agarose gels. Expected product sizes were as follows: FVIII, 276 bp; vWF, 270 bp; VEGF-A, 331 to $350 \mathrm{bp}$; VEGFR2, 350 bp; MMP-9, 433 bp; CXCR4, 200 bp; SDF-1, 245 bp; and $\beta$-actin, $540 \mathrm{bp}$.

PCR analysis. Genomic DNA was extracted with DNeasy Tissue Kit (QIAGEN). Primers for GFP contained in LSECs of FVB/N-Tie2-GFP mice were oIMR1263 5' - ATTCTCGTGGAACTGGATGG-3' and oIMR1264 5'-GGACAGGTAATGGTTGTCTGG-3' (Jackson Laboratory; http://jaxmice.jax.org/strain/003658_2). Platinum PCR Supermix (Invitrogen) was used with 30 cycles at $94^{\circ} \mathrm{C}$ for 3 minutes, $94^{\circ} \mathrm{C}$ for 30 seconds, $60^{\circ} \mathrm{C}$ for 1 minute, $72^{\circ} \mathrm{C}$ for 1 minute, and final elongation at $72^{\circ} \mathrm{C}$ for 7 minutes (Jackson Laboratory; http://jaxmice.jax.org/strain/003658_2). Primers for mouse GAPDH were 5'-GGGTGGAGCCAAACGGGTC-3' (forward) and 5'-GGAGTTGCTGTTGAAGTCGCA-3' (reverse), with 25 cycles at $94^{\circ} \mathrm{C}$ for 3 minutes, $94^{\circ} \mathrm{C}$ for 30 seconds, $56^{\circ} \mathrm{C}$ for 1 minute, $72^{\circ} \mathrm{C}$ for 1 minute, and $72^{\circ} \mathrm{C}$ for 7 minutes, as described (8). PCR products were resolved in $1 \%$ agarose gels. The expected product sizes were 567 (GFP transgene) and $550 \mathrm{bps}$ (GAPDH).

Fluorogenic thrombin generation test. This assay uses a fluorogenic substrate to continuously demonstrate thrombin generation without subsampling or plasma defibrination and is sensitive to less than $0.001 \mathrm{IU} / \mathrm{ml} \mathrm{FVIII} \mathrm{(54).}$ Thrombin generation was initiated by adding $40 \mu \mathrm{l}$ nondefibrinated plasma to $80 \mu \mathrm{l}$ substrate mixture containing $0.238 \mathrm{mM}$ fluorogenic substrate, $5 \mathrm{nMFIXa}, 3 \mu \mathrm{g} / \mathrm{ml}$ phospholipid, and $7 \mathrm{mMCa}^{2+}$ inablack microtiterplate(Greiner Bio-One GmbH). The plate was read in Spectramax Gemini XS Fluorimeter (Molecular Devices) at 30-second intervals for 1 hour at $30^{\circ} \mathrm{C}$ using excitation at $390 \mathrm{~nm}$ and reading at $460 \mathrm{~nm}$. The amount of thrombin generated was quantified for peak thrombin levels and AUC parameters according to the method of Hemker by exporting data into an Excel file (55).

FVIII inbibitor assay. FVIII inhibitor titers were measured using the Bethesda assay (56) with the Nijmegen modification (57). In brief, plasma from test samples was diluted 40-fold with $0.1 \mathrm{M}, \mathrm{pH} 7.4$, imidazole buffer. Equal volumes $(100 \mu \mathrm{l})$ of this and imidazole-buffered C57BL/ 6 mouse plasma also diluted 40 -fold were mixed and incubated for 2 hours at $37^{\circ} \mathrm{C}$.
One-stage FVIII potency assays were then carried out on these samples. A nonspecific antibody (C63 anti-PRL) was used as negative control and an inhibitory/neutralizing antibody (NIBSC reagent $01 / 460$ ) as a positive control. Inhibitory activity was read in Bethesda units/ $\mathrm{ml}$ from semilogarithmic plot representing correlation between residual FVIII:C activity (logarithmic) and inhibitor activity (linear). A minimum of 3 dilutions were made for each test sample.

FVIII antigen ELISA. In brief, 96-well microtiter polystyrene plates (MaxiSorp; Nunc) were coated with $100 \mu \mathrm{l}$ (40-fold diluted) mouse plasma in doubling dilutions in GBS buffer and incubated overnight at $4^{\circ} \mathrm{C}$. After washing $(\times 4)$ with PBS-Tween and saturation (1 hour at room temperature) with $200 \mu \mathrm{l}$ blocking buffer (PBS-HSA, 5\%), $100 \mu \mathrm{l}$ of rabbit A/FVIII polyclonal antibody specific for mouse FVIII (ab53703; Abcam) was added at the manufacturer's recommended concentration (1:5000) in Tris-casein buffer and plates were incubated at $37^{\circ} \mathrm{C}$ for 2 hours. After washing $(\times 3)$ with PBS-Tween, $100 \mu 1$ 1:1000 alkaline phosphatase-conjugated goat polyclonal antibody to rabbit IgG (ab6722-Abcam) diluted in Tris buffer was added to each well and plates incubated at $37^{\circ} \mathrm{C}$ for 1 hours. After further washing, plates were supplemented with $200 \mu \mathrm{l}$ of SIGMA FAST p-nitrophenyl phosphate (pNPP) (Sigma-Aldrich) substrate, and plates were incubated at room temperature in the dark for 30-40 minutes. Once color had developed, the reaction was stopped with $50 \mu 13 \mathrm{M} \mathrm{NaOH}$, and plates were read at $405 \mathrm{~nm}$ on a SpectraMax (Molecular Devices) reader. The amount of FVIII antigen in plasma was measured relative to plasma from healthy C57BL/6 or FVBN mice run concurrently.

Other serological assays. Frozen serum samples were analyzed for alanine aminotransferase, aspartate aminotransferase, bilirubin, and alkaline phosphatase levels with an automated clinical system.

Statistics. Data are shown as means $\pm \mathrm{SD}$. The significance of differences was analyzed by 1 -tailed Student's $t$ test, $\chi^{2}$ test, or ANOVA, where applicable, with SigmaStat software (Jandel Scientific). $P<0.05$ was considered significant.

\section{Acknowledgments}

This work was supported in part by NIH grants R01 DK071111, M01 RR12248, P30 DK41296, P30 CA13330, and CA06576. We are grateful to Chaoying Zhang, Gertrude Arason, and Simone Merlin for technical assistance. A. Follenzi is a recipient of a Liver Scholar Award from the American Liver Foundation/ American Association for Studies of Liver Diseases. We thank R. Sarkar and L. Naldini for critical reviews of the manuscript and for helpful discussions.

Received for publication May 21, 2007, and accepted in revised form December 19, 2007.

Address correspondence to: Sanjeev Gupta, Albert Einstein College of Medicine, Ullmann Building, Room 625, 1300 Morris Park Avenue, Bronx, New York 10461, USA. Phone: (718) 430-2098; Fax: (718) 430-8975; E-mail: sanjvgupta@pol.net.
1. Gupta, S., Inada, M., Joseph, B., Kumaran, V., and Benten, D. 2004. Emerging insights into liver-directed cell therapy for genetic and acquired disorders. Transpl. Immunol. 12:289-302.

2. Do, H., Healey, J.F., Waller, E.K., and Lollar, P. 1999. Expression of factor VIII by murine liver sinusoidal endothelial cells. J. Biol. Chem. 274:19587-19592.

3. Knolle, P.A., and Limmer, A. 2003. Control of immune responses by savenger liver endothelial cells. Swiss Med. Wkly. 133:501-506.

4. Rafii, S., and Lyden, D. 2003. Therapeutic stem and progenitor cell transplantation for organ vascularization and regeneration. Nat. Med. 9:702-712.
5. Lamalice, L., Le Boeuf, F., and Huot, J. 2007. Endothelial cell migration during angiogenesis. Circ. Res. 100:782-794.

6. Griese, D.P., et al. 2003. Isolation and transplantation of autologous circulating endothelial cells into denuded vessels and prosthetic grafts: implications for cell-based vascular therapy. Circulation. 108:2710-2715.

7. Ewing, P., et al. 2003. Isolation and transplantation of allogeneic pulmonary endothelium derived from GFP transgenic mice. J. Immunol. Methods. 283:307-315.

8. Benten, D., et al. 2005. Hepatic targeting of trans- planted liver sinusoidal endothelial cells in intact mice. Hepatology. 42:140-148.

9. Bolton-Maggs, P.H., and Pasi, K.J. 2003. Haemophilias A and B. Lancet. 361:1801-1809.

10. Teitel, J., et al. 2007. A systematic approach to controlling problem bleeds in patients with severe congenital haemophilia A and high-titre inhibitors. Haemophilia. 13:256-263.

11. Lin, Y., et al. 2002. Use of blood outgrowth endothelial cells for gene therapy for hemophilia A. Blood. 99:457-462.

12. Hollestelle, M.J., et al. 2001. Tissue distribution of factor VIII gene expression in vivo--a closer look. 
Thromb. Haemost. 86:855-861.

13. Motoike, T., et al. 2000. Universal GFP reporter for the study of vascular development. Genesis. 28:75-81.

14. DeLeve, L.D., Wang, X., McCuskey, M.K., and McCuskey, R.S. 2006. Rat liver endothelial cells isolated by anti-CD31 immunomagnetic separation lack fenestrae and sieve plates. Am. J. Physiol. Gastrointest. Liver Physiol. 291:G1187-G1189.

15. Nagai, T., et al. 2004. Actin filaments around endothelial fenestrae in rat hepatic sinusoidal endothelial cells. Med. Electron Microsc. 37:252-255.

16. Kumaran, V., Joseph, B., Benten, D., and Gupta, S. 2005. Integrin and extracellular matrix interactions regulate engraftment of transplanted hepatocytes in the rat liver. Gastroenterology. 129:1643-1653.

17. Joseph, B., et al. 2006. Monocrotaline promotes transplanted cell engraftment and advances liver repopulation in rats via liver conditioning. Hepatology. 44:1411-1420.

18. Wu, Y.M., Joseph, B., Berishvili, E., Kumaran, V., and Gupta, S. 2008. Hepatocyte transplantation and druginduced perturbations in liver cell compartments. Hepatology. 47:279-287.

19. Witek, R.P., Fisher, S.H., and Petersen, B.E. 2005. Monocrotaline, an alternative to retrorsine-based hepatocyte transplantation in rodents. Cell Transplant. 14:41-47.

20. Copple, B.L., Banes, A., Ganey, P.E., and Roth, R.A. 2002. Endothelial cell injury and fibrin deposition in rat liver after monocrotaline exposure. Toxicol. Sci. 65:309-318.

21. Deleve, L.D., et al. 2003. Sinusoidal obstruction syndrome (veno-occlusive disease) in the rat is prevented by matrix metalloproteinase inhibition. Gastroenterology. 125:882-890.

22. Malhi, H., et al. 2002. Cyclophosphamide disrupts hepatic sinusoidal endothelium and improves transplanted cell engraftment in rat liver. Hepatology. 36:112-121.

23. Bailey, A.S., et al. 2004. Transplanted adult hematopoietic stems cells differentiate into functional endothelial cells. Blood. 103:13-19.

24. Grunewald, M., et al. 2006. VEGF-induced adult neovascularization: recruitment, retention, and role of accessory cells. Cell. 124:175-189.

25. Kollet, O., et al. 2003. HGF, SDF-1, and MMP-9 are involved in stress-induced human CD34+ stem cell recruitment to the liver. J. Clin. Invest. 112:160-169.

26. DeLeve, L.D. 1996. Cellular target of cyclophosphamide toxicity in the murine liver: role of glutathione and site of metabolic activation. Hepatology. 24:830-837.

27. Bi, L., et al. 1995. Targeted disruption of the mouse factor VIII gene produces a model of haemophilia A. Nat. Genet. 10:119-121.
28. Aronovich, A., et al. 2006. Correction of hemophilia as a proof of concept for treatment of monogenic diseases by fetal spleen transplantation. Proc. Natl. Acad. Sci. U. S. A. 103:19075-19080.

29. Kumaran, V., et al. 2005. Transplantation of endothelial cells corrects the phenotype in hemophilia A mice. J. Thromb. Haemost. 3:2022-2031.

30. Mordenti, J., et al. 1996. Pharmacokinetics and interspecies scaling of recombinant human factor VIII. Toxicol. Appl. Pharmacol. 136:75-78.

31. James, P.D., et al. 2005. Aminoglycoside suppression of nonsense mutations in severe hemophilia. Blood. 106:3043-3048.

32. De Palma, M., et al. 2005. Tie2 identifies a hematopoietic lineage of proangiogenic monocytes required for tumor vessel formation and a mesenchymal population of pericyte progenitors. Cancer Cell. 8:211-226.

33. Shaked, Y., et al. 2005. Genetic heterogeneity of the vasculogenic phenotype parallels angiogenesis; Implications for cellular surrogate marker analysis of antiangiogenesis. Cancer Cell. 7:101-111.

34. Maruyama, K., et al. 2005. Inflammation-induced lymphangiogenesis in the cornea arises from CD11b-positive macrophages. J. Clin. Invest. 115:2363-2372.

35. Lewis, C.E., De Palma, M., and Naldini, L. 2007. Tie2-expressing monocytes and tumor angiogenesis: regulation by hypoxia and angiopoietin- 2 . Cancer Res. 67:8429-8432.

36. Taniguchi, E., et al. 2006. Endothelial progenitor cell transplantation improves the survival following liver injury in mice. Gastroenterology. 130:521-531.

37. Gupta, S., Malhi, H., Gagandeep, S., and Novikoff, P. 1999. Liver repopulation with hepatocyte transplantation: new avenues for gene and cell therapy. J. Gene Med. 1:386-392.

38. Wilson, D.W., Lame, M.W., Dunston, S.K., and Segall, H.J. 2000. DNA damage cell checkpoint activities are altered in monocrotaline pyrroleinduced cell cycle arrest in human pulmonary artery endothelial cells. Toxicol. Appl. Pharmacol. 166:69-80.

39. Shah, M., Patel, K., and Sehgal, P.B. 2005. Monocrotaline pyrrole-induced endothelial cell megalocytosis involves a Golgi blockade mechanism. Am. J. Physiol. Cell Physiol. 288:C850-C862.

40. Kim, K.S., Joseph, B., Inada, M., and Gupta, S. 2005. Regulation of hepatocyte engraftment and proliferation after cytotoxic drug-induced perturbation of the rat liver. Transplantation. 80:653-659.

41. Bontempo, F.A., et al. 1987. Liver transplantation in hemophilia A. Blood. 69:1721-1724.

42. Ohashi, K., et al. 2005. Liver tissue engineering at extrahepatic sites in mice as a potential new therapy for genetic liver diseases. Hepatology. 41:132-140.

43. Jacquemin, M., et al. 2006. FVIII production by human lung microvascular endothelial cells. Blood. 108:515-517.

44. Dasgupta, S., et al. 2007. VWF protects FVIII from endocytosis by dendritic cells and subsequent presentation to immune effectors. Blood. 109:610-612.

45. Chao, H., Monahan, P.E., Liu, Y., Samulski, R.J., and Walsh, C.E. 2001. Sustained and complete phenotype correction of hemophilia B mice following intramuscular injection of AAV1 serotype vectors. Mol. Ther. 4:217-222.

46. Fields, P.A., et al. 2001. Risk and prevention of antifactor IX formation in AAV-mediated gene transfer in the context of a large deletion of F9. Mol. Ther. 4:201-210.

47. Novikoff, P.M., et al. 1996. Three-dimensional organization of rat hepatocyte cytoskeleton: relation to the asialoglycoprotein endocytosis pathway. J. Cell Sci. 109:21-32.

48. Chao, H., et al. 2003. Phenotype correction of hemophilia A mice by spliceosome-mediated RNA trans-splicing. Nat. Med. 9:1015-1019.

49. Wu, T., Handa, J.T., and Gottsch, J.D. 2005. Lightinduced oxidative stress in choroidal endothelial cells in mice. Invest. Ophthalmol. Vis. Sci. 46:1117-1123.

50. Chen, S.D., et al. 2005. Pravastatin attenuates ceramide-induced cytotoxicity in mouse cerebral endothelial cells with HIF-1 activation and VEGF upregulation. Ann. N. Y. Acad. Sci. 1042:357-364.

51. Brekken, R.A., et al. 2003. Enhanced growth of tumors in SPARC null mice is associated with changes in the ECM. J. Clin. Invest. 111:487-495.

52. Wang, F.S., et al. 2003. Population survey of CCR5 delta32, CCR5 m303, CCR2b 64I, and SDF1 3'A allele frequencies in indigenous Chinese healthy individuals, and in HIV-1-infected and HIV-1-uninfected individuals in HIV-1 risk groups. J. Acquir. Immune Defic. Syndr. 32:124-130.

53. Molyneaux, K.A., et al. 2003. The chemokine SDF1/ CXCL12 and its receptor CXCR4 regulate mouse germ cell migration and survival. Development. 130:4279-4286.

54. McIntosh, J.H., Owens, D., Lee, C.A., Raut, S., and Barrowcliffe, T.W. 2003. A modified thrombin generation test for the measurement of factor VIII concentrates. J. Thromb. Haemost. 1:1005-1011.

55. Hemker, H.C. 1999. Endogenous thrombin potential. In Laboratory techiques in thrombosis: a manual. J. Jespersen, R.M. Bertina, and F. Haverkate, editors. Kluwer Academic Publishers. Dordrecht, The Netherlands. 63-79.

56. Kasper, C.K., et al. 1975. Proceedings: A more uniform measurement of factor VIII inhibitors. Thromb. Diath. Haemorrh. 34:612.

57. Verbruggen, B., et al. 1995. The Nijmegen modification of the Bethesda assay for factor VIII:C inhibitors: improved specificity and reliability. Thromb. Haemost. 73:247-251. 